adult flagellate stage they have the same general morphological build. They all undergo their developmental stages in the intestinal tracts of insects, but the fact that one of them passes its pre-flagellate stage in man has led most observers to regard it as something quite distinct from a Iterpetomonas. The parasite of kala azar is certainly not a sporozoon; it remains throughout its life-cycle as far as we know it a typical flagellate. With a complete knowledge of the life-cycle of such a closely allied form as Herpetomonas lygaei, it is possible to conjecture that the parasite of kala azar in order to get back to man must pass back again to a non-flagellate stage, post-flagellate, and that this change is most likely to take place somewhere in the neighbourhood of the biting parts of the bed bug. Had I known the complete life-cycle of Herpetomonas lygaei when I had $m$ y heavily infected bugs I might have been able to solve this problem. This further emphasises the importance of studying seemingly unimportant parasites, and I am sorry to say that there are some people who still think it is a waste of time to study such forms, but there is no doubt that a knowledge of their life-cycles is of the utmost importance in helping to explain those of more complex pathogenic protozoa.

I have now shown you that the parasite of kala azar is a herpetomonad and that in its pre-flagellate stage it may be indistinguishable from a harmless species; this truth is of importance, because in spite of other data the two closely allied human forms - the parasites of infantile splenomegaly and oriental sore-are still regarded as identical with the parasite of kala azar. I know at least three closely allied herpetomonads which are very similar in their pre-flagellate stages to Herpetomonas musce domestice, so that this similarity in one stage is of little importance in distinguishing between these species. The parasite of kala azar should, therefore, be known as Herpetomonas donovani (Laveran and Mesnil), the parasite of infantile splenomegaly as Herpetomonas infantum (Nicolle), and the parasite of oriental sore as Herpetomonas tropica (Wright), and I would suggest that the diseases produced by these parasites should be collectively known as herpetomoniases.

It is most unfortunate that all attempts to transmit Herpetomonas donovani to animals have entirely failed; quite recently I inoculated dogs with the parasite with negative results. It seems to me, therefore, that it will be well-nigh impossible to prove finally that Cimex rotundatus is the carrier of this dangerous parasite. It is to be hoped that exhaustive feeding experiments similar to those I carried out in Madras will be conducted with Herpetomonas infantum and Herpctomonas tropica. However, I feel bound to say here that there is too great a tendency at present to cultivate these parasites in test-tubes ; these experiments are undoubtedly highly interesting, yet they will not give us the clue as to how the parasites are transmitted from man to man. Feeding experiments with blood-sucking insects in the case of these herpetomonads are very difficult and tedious owing to the fact that unless a very good case is selected it is almost impossible to follow one or two developing parasites in the guts of the insects. Further, such experiments must be carried out with great precision, and the observer must possess a good working knowledge of the many harmless gutflagellates which occur in nearly every insect. So far as I am aware no attempts have been made to discover the transmitting agent of Herpetomonas infantum or Herpetomonas tropica.

\section{A NOTE ON THE TREATMENT OF SYPHILIS. ${ }^{2}$}

BY G. G. STOPFORD TAYLOR, M.D. DURH., PHYSICIAN TO THE IIVERPOOL SEIN HOSPITAL.

IT is recognised that mercury is the antidote for syphilis in the primary and secondary stages, while iodide of potassium is usually prescribed alone in the tertiary stage, or combined with or given alternately with mercury. The method of administering mercury which $I$ practise and advocate, though not to the exclusion of other methods, is that of the intramuscular injection of grey oil which contains 40 per cent. by weight of metallic mercury. It has many

1 Part of a post-graduate lecture delivered at the Liverpool skin Fospital. advantages over other methods, inasmuch as it is scientific, as the physician administers the dose himself, and therefore knows the exact amount which his patient is receiving, and as it is necessary to repeat these injections weekly the progress made by the patient may be observed and the physician is prepared for any complication or emergency which may arise.

Contradictory opinions have been expressed as to the pain caused by this method. All injections must necessarily cause a certain amount of pain, though in our practice it is rare to have any complaints. If we inquire, the patient will admit a certain amount of stiffness coming on two or three days afterwards. After a few injections, however, he gets quite accustomed to them and will tell you that he prefers the treatment to the everlasting pills and mixtures. Occasionally it happens that he will state before leaving the consulting-room that the last injection caused more pain at the moment than any previous injection. It is difficult to explain this unless we can assume that the needle has wounded a nerve. In four and a half years I have only found it necessary in one case to discontinue intramuscular injections with grey oil because of pain, and I am unable to explain why the same patient suffered as he said and why he submitted without a murmur to intramnscular injections of the sozoiodolate of mercury twice weekly into his deltoid muscles. Our experience in hospital practice is the same. No greater testimony to the vaiue and comparative painlessness of the injections of grey oil can be adduced than the fact that syphilitic derelicts attend most regularly, and they ought to know. I mean by derelicts those who have wandered from practitioner to practitioner and from hospital to hospital and have experienced all methods of treatment without being cured.

Site of injection. - The gluteal region is by preference the most suitable because of the size and thickness of the muscles in this locality, but it is necessary to choose a spot sufficiently far removed from the great sacro-sciatic notch so as to avoid wounding any of the large vessels and nerves emerging therefrom. This is a point midway between the upper end of the gluteal furrow and the anterior superior spine of the iliac crest.

Method of injection.-The skin having been well and thoroughly cleansed with methylated spirit, the butt end of the needle is held firmly between the finger and thumb of the right hand and with a keen eye and a steady hand is quickly plunged into the buttock, with the point of the needle very slightly inclined outwards. It is well to wait 30 seconds to see if any blood flows from the needle before injecting the oil, or, better still, to exhaust it with an ordinary hypodermic syringe. The oil is then slowly injected and when the operation is completed the needle is again very quickly withdrawn so as to prevent, if possible, any of the mercury escaping from the muscle along the needle track. The part is then massaged for 30 seconds. No dressing is required.

The position of the patient during the operation is of importance. I always place him in a stooping posture, heels together, leaning with his hands on the end of a couch or chair to steady himself. The introduction of the needle is done so quickly that only the slightest prick is felt. No patient has ever fainted.

Bleeding.- Should any bleeding follow the insertion of the needle it must be withdrawn and re-inserted in another place. A drop or two of blood sometimes appears as the needle is withdrawn; this, however, is controlled by a few moments' pressure.

The dose of mercury given at each injection depends, first, upon the weight, activity, and habits of the patient; and secondly, upon his power of elimination of the drug by the kidneys. For an active man of from 12 to 14 stones in weight and in the early stages of the disease I prescribe 14 centigrammes. For a less active man, say of from 10 to 12 stones, eight centigrammes. For a woman usually seven centigrammes, though this is exceeded in urgent cases for the first three or four injections. From 10 to 12 injections constitute a course, after which a rest of two months is taken and then a repetition of the treatment is begun. Three courses a year for two years are my minimum; afterwards one or two courses annually according to circumstances.

The syringe which I use is that of Barthelemy; it is graduated in centigrammes, a complete syringeful holding in all 14 centigrammes, and as this amount of grey oil 
contains $2 \cdot 5$ grains of metallic mercury by weight no mistake in the dose can be made. We prefer to use platinum needles. It is true that they are expensive and that the points soon become blunt with use, but the advantages are that they do not corrode under the influence of the mercury and therefore are easily sterilised. The length of the needles is one and three-quarter inches for general use, varying to suit the obesity of the patient.

The objections raised to this method of administering mercury are several, but it is a remarkable fact that the most strenuous opposition has proceeded from those members of the profession who have never tried it. The objections are abscesses, nodes, embolism or thrombosis, and fatal salivation. After four years' daily experience of this method we have never had or seen a case of abscess. In our early days we had a few nodes amongst hospital patients, but this was due to faulty technique, which we believe we have remedied by cleansing the skin with methylated spirit in preference to other antiseptics, and by using greater judgment in choosing needles of such a length as will place the oil deeply in the muscles, as a node can only form when the mercury remains immediately beneath the skin.

Subcutaneous nodes arising after injection of grey oil can only occur through some defect in the technique of the operation. This is confirmed by the fact that though $I$ have given some hundreds of injections $I$ have never had an accident amongst $m y$ private patients, but they have occurred in the practice of others, as I have witnessed, and as has been reported in the journals from time to time. Such an accident, if frequently repeated, is sufficient to condemn the method of intramuscular injections. The question arises, How are they to be avoided? First, by extreme cleanliness; and secondly, by using a sufficiently long needle and by taking care that it is pushed well home into the muscle on pushing down the piston of the syringe and thereby insuring that none of the oil has been left in the subcutaneous tissue, or otherwise a node, followed may be by an abscess, is bound to occur. If in spite of these precautions nodes have still occurred they have been due to faulty needles.

In order that what I mean by the word "faulty" may be understood I must draw attention to the manufacture of the needles Steel needles are drawn as wire is. Platinum needles we used to believe were also drawn, but there is reason to think that the latter are rolled and the seams soldered, Now, solder consists of lead and tin, or silver and tin, and is rapidly eroded by mercury, whilst platinum and iridium are not; consequently, minute perforations occur through the solder by which the grey oil escapes into the subcutaneous tissue. These perforations may occur at any part in the shaft of the needle. A slit was discovered by Dr. R. W. Mackenna close to the mount; others were observed by myself close to the point. Now i believe these perforations occurred through not cleansing the needle immediately after it was used. "Faulty" platino-iridium needles have not as yet been given as a reason for nodal formation. have used some of my platino-iridium needles for four years and have always cleansed them at once, and therefore $I$ am convinced that owing to this precantion I have escaped the accidents previously referred to. I never use steel needles as they become eroded and are apt to break.

Embolism or thrombosis we have never seen. Slight salivation has occurred in a few hospital patients because of the unhealthy condition of their gums. In the cases of fatal salivation which occurred among the troops in the Egyptian command in 1901 I am of opinion that they arose, first, because the doses were too large and administered too frequently, and also that the patients were delilitated from alcoholism, dysentery, and malaria. ${ }^{2}$ Smaller and less frequent doses are now recommended by the Armp Medical Department and such is its confidence in the method that one and a half pounds of mercurial cream are supplied every week to the various military centres.

No matter what method may be adopted salivation may arise, but it rarely occurs in my private practice because I always have the patient's mouth attended to. In patients of the hospital class, the majority of whom have defective teeth and have never used a toothbrush in their lives, it occasionally happens as I have said, although they are supplied with mouth washes and tooth powders. Fournier gives four chief types of salivation: (1) inferior median gingivitis which affects the lower incisors; (2) peripheral

\footnotetext{
2 See $\Lambda$ rmy Reports.
}

gingivitis which affects a carious tooth or stump; (3) genial stomatitis affecting the mucous membrane of the cheek opposite the last lower molar ; and (4) retro-molar detachment. This consists in the separation of the gum behind the last molar tooth which bleeds easily.

Caution. - Now it may happen that a patient is under treatment who at the same time is cutting a wisdom tooth and the symptoms arising from this being similar may lead his medical attendant astray.

The following is Lafay's formula of grey oil: mercury, 40 grammes; sterilised lanoline, 12 grammes ; white vaseline, 13 grammes; and medicinal oil of vaseline, 35 grammes.

Intramuscular injections. - When the mercury is deposited in the muscles the absorption is very slow, as may be seen by radiographs taken from time to time. Some investigators have recorded that it has disappeared in a few days, but from radiographs taken by Dr. W. C. Oram and Dr. Mackenna in our laboratory mercurial shadows have been distinctly observed three weeks after the injection. Naturally, therefore, a good deal depends upon the radiographer and the apparatus. Having placed the mercury in the patient's gluteal muscles and seen it there we may now inquire how it is got rid of. Mercury may be said to be eliminated by three channels-viz, by the mucous membranes of the mouth, by the mucous membranes of the intestines, and by the kidneys, and therefore it is essential that these parts should be in a healthy condition, for if from any cause these emunctories are diseased trouble is almost certain to occur. For instance, ptyalism when the gums are unhealthy, diarrhœa if the patient is subject to looseness of the bowels or has had dysentery, and profound mercurial poisoning if the kidneys are diseased. It is, therefore, essential that in all cases and in all methods of administering mercury, the urine should be examined at intervals, so that we may know how the kidneys are doing their work and if the specific gravity is below 1015 we must proceed cautiously. The average time in which mercury has been found in the urine after a single injection is one week, though we cannot say that elimination has nc's been taking place before that, perhaps from the bowels, for we have one patient whose stools become more and more frequent in 12 hours after each injection and continue so for several days after each injection and therefore, we never repeat an injection until his bowels have resumed their normal condition.

Analysis of urine for mercury conducted in my laboratory by Dr. Mackenna and Dr. Oram in 1906 and 1907-08 gave an average of from two and a half to three months as the latest periods in which mercury can be found after cessation of treatment, but this depends upon the length of the course.

The advantages of this method of treatment are convincing to my mind : (1) accurately measured doses of mercury are deposited in the muscles at regular intervals which undergo slow absorption and elimination, during which periods the blood and tissues in which the spirochætæ swarm are mercurialised-or, in other words, the spirochæta is in a mercurial bath; (2) the stomach is reserved for food; and (3) during the courses of treatment the patient is obliged to visit his medical attendant and is thus kept under control

The detection of meroury in the urine. - The test for mercury in the urine is a long and laborious process and quite outside the work of a general practitioner. Fortunately, we can do without it, for if a 24 hours sample of urine is not below specific gravity of 1015 and is free from albumin and sugar we may take it for granted that the renal organs are working well.

Iest for mercury (detection according to Stukowenkoff). Five cubic centimetres of egg albumin are thoroughly rubbed in a mortar with an equal quantity of a saturated solution of sodium chloride and dissolved in 500 cubic centimetres of urine. The solution is then warmed on a water-bath until the albumin is completely coagulated. The precipitate is collected on a filter, dried between filter paper, and then rubbed in a mortar with about 10 cubic centimetres of concentrated hydrochloric acid. 40 cubic centimetres of hydrochloric acid are then added and the solution, in which a copper or brass spiral is ulaced, is allowed to stand 24 hours in a glass beaker or cylinder. The albumin and the mercury which has been collected by it are dissolved by the hydrochloric acid. The mercury forms an amalgam on the surface of the copper spiral. The spiral is washed first with cold and then with hot water, rinsed in alcohol and ether, and dried in the air. It is placed in a dry piece of narrow glass tubing which has been sealed at one end by melting. 
A few crystals of iodine are then placed in the tube and the tube is carefully heated with continuous turning from the lower to the upper end of the copper spiral. The mercury is thus sublimed, and, combining with the iodine, forms a brick-red ring of mercuric iodide. The width of the ring is, if the instructions are carried out, exactly proportional to the quantity of mercury, and a quantitative estimation is thus rendered possible. It is only necessary to have a scale, that is, a series of mercuric iodide rings obtained from definite quantities of mercury $(1,2,3$, 4 , \&c., milligrammes), and to compare the ring obtained with this scale. This method is very delicate; 0.0005 gramme of mercury can be clearly detected. If the ring is not clearly seen macroscopically the characteristic red crystals of mercuric iodide can be easily detected microscopically with a low power.

Finally, intramuscular injections are not as a rule suitable for children, neither are mercurials of any kind or form beneficial in parasyphilitic diseases such as locomotor ataxia and general paralysis; but in saying this one must differentiate between true syphilitic lesions of the brain and spinal cord, where they are of the greatest value.

Strict attention must be paid to the hygiene of the mouth. The teeth are to be brushed night and morning and the mouth rinsed very frequently during the day with a solution of acetate of alum: one ounce of alum with five ounces of distilled water, and one ounce of subacetate of lead with five ounces of distilled water. Mix the solutions and filter. For use dilute with 19 parts of water.

Ireatment by inunction.- The mercury is to be absorbed by the skin as well as by inhalation of the volatilised metal. Inunction was one of the earliest methods of treating syphilis, but it fell into disrepute owing to the reckless way in which it was used. At Aachen, where this method is carried out systematically, at 6.30 A.M. the patient drinks three glasses of hot spring water; at 7.30 he has a hot bath which is followed by inunction, and breakfast is provided at 8.30. Afterwards the patient rises and takes light exercise in the open air till lunch at 1.30 P.M. More exercise is taken afterwards and three glasses of bot spring water are drunk. Dinner is served at from 6 to 7 P.M., the patient retiring to bed at 10 P.M. Three grammes of mercurial ointment containing 15 grains of metallic mercury are rubbed into the skin of the calves, the thighs, the back, the chest, and the arms for 20 minutes exactly by specially trained men. The change of site is to avoid irritation of the skin.

Another method is to spread from half a drachm to one drachm of mercurial ointment upon the abdomen before retiring to rest and to cover with a bandage or binder. On the next morning the skin is washed clean with soap and water. This is an excellent plan for treating congenital syphilis in infants, though of course a less dose diluted with lard is necessary.

The advantages claimed for inunction are $(1)$ that there is no interference with the digestive functions, and (2) its painlessness. The objections are (1) that it is dirty and has sometimes excited attacks of acute dermatitis and (2) that it requires specially trained rubbers and for obvious reasons cannot be carried out in the homes of patients in this country.

Methods of administering mercury by the mouth. - The preparations usually employed are :- Hyd. cum cret. with or without Dover's powder ; liq. hyd. bichlor.; pil. hyd. 3 grains pil. Plummer. The disadvantages are: (1) that absorption is irregular; (2) that in sıme patients it appears to have no visible effect upon syphilitic lesions ; (3) serious disturbance of the alimentary tract; and (4) that the average duration of treatment is two years.

Diet.-Generous diet is necessary as waste of tissue is increased, avoiding green vegetables, fruit, and spices which may cause irritation as the mercury renders the gastro-intestinal tract more sensitive.

Levy Bing states that when mercury is administered to syphilitic patients, the excretion of urea, the percentage of hæmoglubin, and the body weight are all increased. By noting one or more of these points during a course of treatment a certain amount of information may be obtained as to wherher to continue mercury or not, an increase being an indication to continue and a decrease the reverse.

I conclude by a résumé of the special indications for the injection method. 1. When a rapid therapeutic effect is desired. 2. In cases of syphilis of the central nervous system. 3. In hot climates, when the gastro-intestinal system is more liable to be upset by the oral administration of mercury.

Soluble salts. -Advantages : any desired quantity of mercury can be introduced daily and is rapidly absorbed; its effects can be watched and the dose modified to suit each case. The special indications for soluble salts are: (1) tuberculous cases; (2) in cases of extensive caries; (3) in cases in which the liver and kidneys are not acting well; (4) in young children who tolerate injections of soluble salts better; (5) in eye cases; and (6) in debilitated people. Disadvantages : The great objection is the necessity of daily administrations, resulting in waste of time to the surgeon and the patient and a frequent recurrence of more or less pain.

Insoluble salts. - The object in using insoluble salts is to introduce into the system at definite intervals a quantity of mercury which shall act as a supply depôt and be gradually but continually absorbed. Advantages : (1) fewer injections ; (2) no waste of time; and (3) inexpensive. Disadvantages : (1) should mercurial poisoning occur it is impossible to get rid of the mercury in the body (this rarely happens if the method is carefully carried out) ; (2) grey oil causes pain, but very little; (3) abscess is generally the result of sepsis ; (4) the nodes are usually small and not painful and are due to subcutaneous injections; and (5) inflammatory reaction (this only occurs after the use of calomel, never after grey oil). Liverpool.

\section{ON A "TYPHOID CARRIER," TREATED SUCCESSFULLY BY THE INOCULA- TION OF' TYPHOID VACCINE.'}

BY S. T. IRWIN, M.B., M.CH. R.U.I., SURGICAL REGISTRAR, ROYAI VICTORIA HOSPITAL, BELFAST; $A N D$

THOMAS HOUSTON, M.D. R.U.I., JOINT LECTURER IN MEDICAI JURISPRUDENCE, QUEEN'S COLLEGE, BELFAST, ETC.

(From the Queen's College Pathological Laboratory.)

THE essential points in the history of this case are as follows. The patient, aged 26 years, while a servant in a house in Liscard, contracted typhoid fever seven years ago and was confined to bed for seven weeks. The girl at first stated that her recovery from this illness was complete and she was soon able to return to work. When questioned, however, she admitted that since her illness she had not been in robust health. She stated that she was easily tired, that she suffered from frequent headaches and occasional gastric disturbances. Her general condition evidently varied but her health was not altogether satisfactory. After her recovery from this acute illness she took another situation in Liscard, and after she was one month in this place the gentleman of the house developed typhoid fever about the end of March or the beginning of April. For the following two years she was in different situations but nothing of importance occurred. She then took a situation in Liverpool where the son of her mistress developed typhoid fever in March or April. Finally, she came to Belfast and while in service in this city four people in the same house developed typhoid fever at short intervals (June to July). In other words, in the course of seven years six people living in the same houses as the patient developed typhoid fever. One of us suspecting that she might be a " typhoid carrier" had her blood examined. It was found to give an imperfect Widal reaction in 1 in 50 dilution in one hour and a good reaction in lower dilutions. She was therefore advised to go into hospital that her condition might be investigated. She was admitted to the medical wards of the Royal Victoria Hospital on July 18th, 1908, and was placed under the care of Dr. W. Calwell who kindly allowed us every opportunity to investigate the case. A catheter specimen of her urine was obtained; it was

1 A paper communicated to a meeting of the Belfast division of the British Medical Association on Dec. 16th, 1908. 